\title{
STABILITY ENHANCEMENT OF A SUPERCONDUCTING GENERATOR USING AN OPTIMIZED SVC-BASED STABILIZER
}

\author{
R. A. F. Saleh \\ Electrical Engineering Department \\ Faculty of Engineering, Menoufiya University
}

\begin{abstract}
An important aspect in developing superconducting generator (SCG) concerns stability following a major system disturbance. This paper presents a method for enhancing stability of a SCG connected to an infinite-bus system using one of FACTS devices. In this method, a static VAR compensator (SVC)-based stabilizer is designed in coordination with a governor controller (GC) to effectively damp the mechanical oscillations which arise in the system when subjected to a disturbance. A time response-based objective function is defined and the design problem of SVC-based stabilizer and GC is formulated into an optimization problem. Particle swarm optimization (PSO) technique is employed to find out an optimal set of parameters for SVC-based stabilizer and GC. Simulation results, damping torque analysis, and small signal analysis show that the proposed PSO-based control scheme provides more damping to the SCG, and enhances its stability over a range of operating conditions.

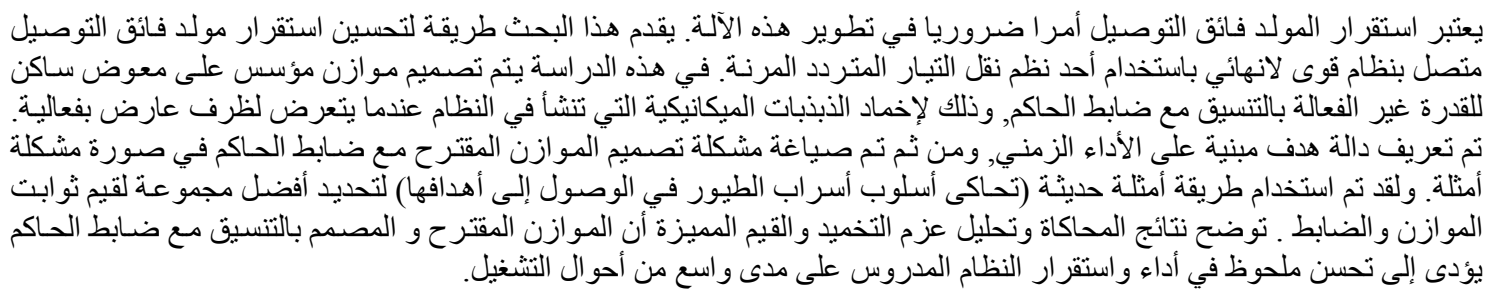

Keywords: Superconducting generator, Transient stability, FACTS, Particle swarm optimization

\section{Introduction}

Superconducting generators have several potential advantages such as small size, light weight, high efficiency and increased steady state stability limit [1-2]. The advantages of SCG have drawn more interest in industrial countries since 1970's, such as in USA, UK and Japan where many R\&D projects on SCGs have been conducted at utility companies, power plant manufacturers and other organization toward a 200 MW class pilot-machine [3-7]. However, superconducting generators also have characteristics that degrade their stability when connected to the power system. Moreover, the very long field winding time constant and the shielding effect of the two rotor screens make the achievement of acceptable dynamic performance very difficult using excitation control. Governor control hence becomes the only technique feasible for stability enhancement of the SCG. The availability of electrohydraulic governors and fast operation of steam valves has now made it possible to obtain very fast turbine response. Research works reported in Ref. [8-9] have shown that SCG stability can be improved by introducing a phase advance network (conventional stabilizer) in the governor feedback loop, activated by the speed error signal. The conventional stabilizer parameters are fixed to ensure optimum performance at a specific operating point. However, because of the high nonlinearity of the machine/power system combination, the stabilizer's performance becomes lower when the system operating condition moves significantly away from the specific point. Therefore, the conventional stabilizer should have some degree of robustness to be able to stabilize the system over a wide range of operating conditions. Many attempts along with comprehensive analysis have been made to improve matters a) by retuning the conventional stabilizer, b) by utilizing adaptive control technique and c) by adopting a fuzzy logic stabilizer [10]. In all these attempts, stabilizers' parameters were selected using genetic algorithm (GA) technique.

Recently, the flexible AC transmission systems (FACTS) have been introduced, in which various power electronics-based controllers are used to maximize the utilization of transmission assets efficiently and reliably [11-12]. In addition, FACTS devices regulate power flow and, through rapid control actions, can mitigate low frequency oscillations and enhance power system stability [1314]. A literature survey on the work done on the application of FACTS devices along with the excitation control to enhance damping of conventional generator oscillations is given in the introduction of Ref. [14].

Early investigation on the dynamic performance of a SCG when equipped with a static VAR compensator 
at its terminal was reported in Ref. [15]. In that study, the stabilizing signal was not optimized. Moreover, the governor role in damping the machine oscillation was not considered. However, no or little efforts have been made towards stability enhancement of SCG using coordinated governor controller and FACTS device-based stabilizer. In this paper, enhancement of SCG stability using coordinated design of a static VAR compensator (SVC)-based stabilizer and a governor controller (GC) is studied. The optimal parameters of SVCbased stabilizer and GC are sought by utilizing the PSO technique [16]. Non-linear simulation and small signal analysis are carried out to investigate the effectiveness of the proposed scheme.

\section{System Description}

The system considered in this study is a SCG connected to an infinite bus power system as shown in Fig. 1. The SCG has superconducting field windings in the rotor, surrounded by two separate screens. The inner screen, which has a relatively long time constant, shields the superconducting field windings from external, time varying magnetic fields. The outer screen serves as a damper and has a substantially shorter time constant than that of the inner screen [17]. The SCG is driven by a threestage steam turbine with reheat between the high pressure and intermediate pressure stages. The turbine is controlled by fast acting electro-hydraulic governors fitted to the main and interceptor valves, which are working in unison. The system is equipped with a governor controller and a SVC at the terminal of the SCG. The exciter voltage, $U_{e}$, of the SCG is kept constant during transients.

\section{Mathematical Model}

The mathematical models for SCG, turbine and governor are shown below, while the parameter values and physical constraints are given in Appendix A.

\subsection{Superconducting generator model}

Based on Park's $d-q$ axis representation, seven nonlinear differential equations are used to represent the mathematical model of the SCG's electric circuits. These equations along with the mechanical equations of motion give the flux linkage model of the SCG [9] as follows:

$$
\begin{aligned}
& p \psi_{d}=\omega_{o}\left[V_{d}+i_{d} R_{a}+\psi_{q}\right]+\psi_{q} \omega \\
& p \psi_{q}=\omega_{o}\left[V_{q}+i_{q} R_{a}-\psi_{d}\right]-\psi_{d} \omega \\
& p \psi_{D 1}=-\omega_{o} i_{D 1} R_{D 1} \\
& p \psi_{Q 1}=-\omega_{o} i_{Q 1} R_{Q 1} \\
& p \psi_{D 2}=-\omega_{o} i_{D 2} R_{D 2} \\
& p \psi_{Q 2}=-\omega_{o} i_{Q 2} R_{Q 2} \\
& p \psi_{f}=\omega_{o}\left[V_{f}-i_{f} R_{f}\right] \\
& p \delta=\omega \\
& p \omega=\frac{\omega_{o}}{2 H}\left[T_{m}-T_{e}\right] \\
& T_{e}=\psi_{d} i_{q}-\psi_{q} i_{d}
\end{aligned}
$$

where:

$P \quad$ : derivative operator

$\psi \quad$ : flux linkage

$\omega_{0} \quad$ : synchronous speed $(\mathrm{rad} / \mathrm{s})$

$\omega$ : rotor speed deviation from $\omega_{0}$

$\delta \quad$ : rotor angle with respect to infinite bus

$H \quad$ : inertia constant

$T_{m} \quad$ : mechanical torque

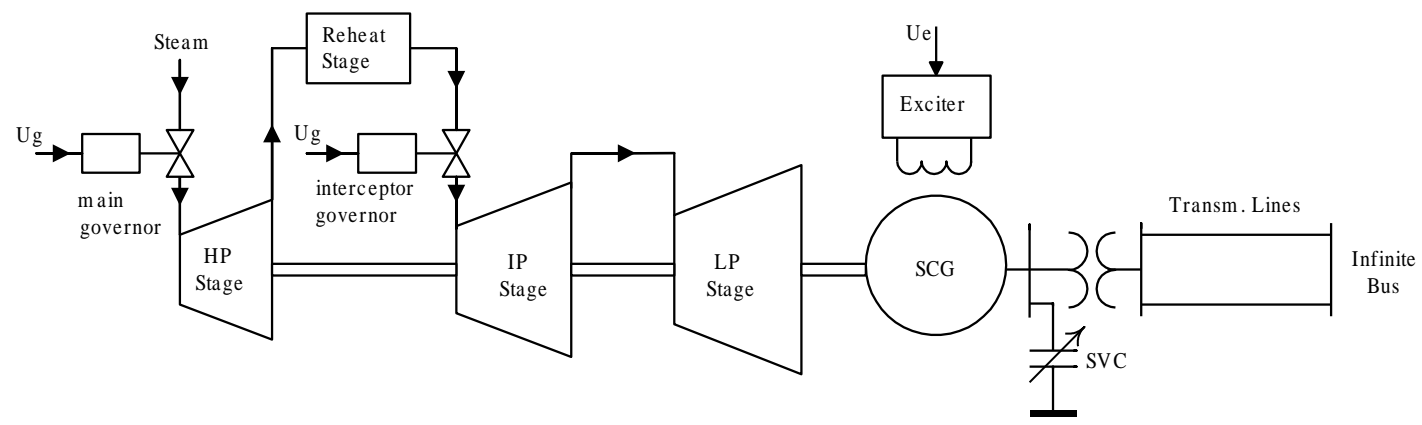

Fig.1 SCG system under study with SVC 


\subsection{Turbine and governor model}

The mathematical model of the turbine and governor system is represented by six nonlinear differential equations [18] as follows:

$$
\begin{aligned}
& p Y_{H P}=\left(G_{M} P_{o}-Y_{H P}\right) / \tau_{H P} \\
& p Y_{R H}=\left(Y_{H P}-Y_{R H}\right) / \tau_{R H} \\
& p Y_{I P}=\left(G_{I} Y_{R H}-Y_{I P}\right) / \tau_{I P} \\
& p Y_{L P}=\left(Y_{I P}-Y_{L P}\right) / \tau_{L P} \\
& p G_{M}=\left(U_{g}-G_{M}\right) / \tau_{G M} \\
& p G_{I}=\left(U_{g}-G_{I}\right) / \tau_{G I}
\end{aligned}
$$

The output mechanical torque is given as:

$T_{m}=F_{H P} Y_{H P}+F_{I P} Y_{I P}+F_{L P} Y_{L P}$

where:

$$
\begin{array}{ll}
P_{o} & : \text { boiler steam pressure } \\
Y & : \text { output of a turbine or reheat stage } \\
\tau & : \text { time constant of stage }
\end{array}
$$

$G_{\mathrm{M}}, G_{\mathrm{I}}:$ main and interceptor valve positions $F_{H P}, \quad F_{I P}, \quad F_{L P}:$ fractional contributions of turbine stages into $T_{m}$

\section{$U_{g} \quad$ : governor actuating signal}

The main and interceptor valves are conventionally actuated by a normalized speed error signal incorporating a droop, typically $4 \%$. Constraints are imposed on valve positions and rates of movement. The rate constraint is based on complete opening or closing time for the valves of $150 \mathrm{~ms}$. The rate limits correspond to the fastest valve operation reportedly available in literature [18].

\section{The Proposed Approach \\ 4.1. Control objective}

The control objective is to generate two stabilizing signals using the speed error signal. The first control signal is produced via a conventional controller and then introduced into the governor loop of the SCG system as shown in Fig. 2. The control signal, $u$, generated by the conventional controller is given as:

$$
u=G_{s} \cdot \frac{\left(1+T_{1} s\right)}{\left(1+T_{2} s\right)} \cdot \omega
$$

where $\omega$ is the speed error signal, $G_{s}, T_{1}$ and $T_{2}$ are the controller parameters, which have to be designed properly to achieve a satisfactory performance.

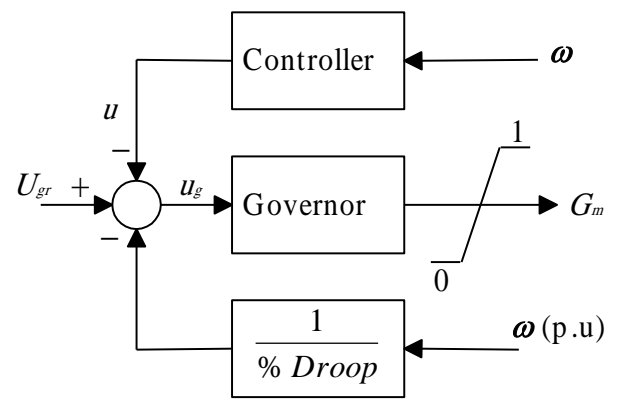

Fig.2 The governor control system

The second signal is produced via a SVCbased conventional lead stabilizer. The two stabilizing signals are coordinated to enhance the damping of the rotor oscillations after disturbances, and hence to improve the transient and dynamic performance of the system.

\subsection{SVC-based stabilizer}

The block diagram of an SVC with a conventional lead stabilizer is shown in Fig. 3. Functionality, the SVC is thought of as an adjustable shunt susceptance that can be varied with sufficient rapidity. Elaborated model for SVC can be seen in Ref. [19]. However, the susceptance, $B$, of the SVC can simply be expressed as:

$$
p B=\left(K_{s v c}\left(B_{r e f}+u_{S v C}\right)-B\right) / T_{s v c}
$$

where $K_{s v c}$ and $T_{s v c}$ are the gain and time constant of the SVC. $B_{r e f}$ is the reference susceptance of the SVC and $u_{S V C}$ is the stabilizing signal generated by the conventional stabilizer installed in the feedback loop of the SVC as shown in Fig. 3.

$$
u_{S V C}=K_{v} \cdot \frac{\left(1+T_{3} s\right)}{\left(1+T_{4} s\right)} \cdot \omega
$$

where $K_{v}, T_{3}$ and $T_{4}$ are the SVC-based stabilizer parameters, which need a careful selection to enhance the system stability. Both of $u$ and $u_{S V C}$ has upper and lower limits, i.e.

$$
u_{\min } \leq\left(u, u_{S V C}\right) \leq u_{\max }
$$

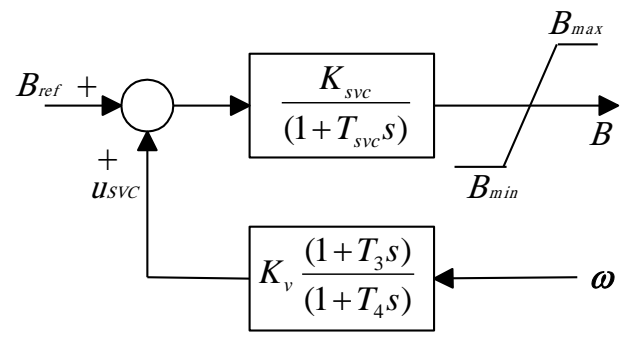

Fig.3 SVC with lead stabilizer 


\section{Stabilizer Parameters Selection Using PSO}

Recently, a heuristic search method called particle swarm optimization (PSO) has been introduced [20]. PSO is characterized as a simple concept, easy to implement, and computationally efficient. Theses features make PSO technique able to accomplish the same goal as GA optimization in a new and faster way. A number of very recent successful applications of PSO on various power system problems have been reported in literature [16]. The tuning parameters in the proposed approach are $G_{\mathrm{s}}, T_{1}$ and $T_{2}$ for GC, and $K_{v}, T_{3}$ and $T_{4}$ for SVC-based stabilizer. Usually, $T_{2}$ and $T_{4}$ are prespecified leaving the other four parameters, $G_{\mathrm{s}}, T_{1}, K_{v}$ and $T_{3}$ to be tuned $[14,21]$. Here, the degree of freedom in the design problem is increased by letting $T_{2}$ and $T_{4}$ be freely selected as well as the other four tuning parameters. This addition is intended to enhance the effectiveness of the proposed stabilizer. Therefore, we have now six parameters to be optimally chosen. This task is achieved using the PSO technique. To do so, the following quadratic performance index, $J$, is first defined.

$$
J=\sum_{k=1}^{N}\left\{\left[k T_{s} . \omega(k)\right]^{2}+[\Delta \delta(k)]^{2}+\left[\Delta G_{M}\right]^{2}\right\}
$$

where $\Delta \delta(k)=\left(\delta(k)-\delta_{o}\right)$ denotes the deviations (in radians) of the instantaneous rotor angle from its steady state value, $\delta_{o}$, and $\Delta G_{M}(k)=($ $\left.G_{M}(k)-G_{M o}\right)$ is the deviation of the instantaneous governor valve position $G_{M}(k)$ from its value in the steady state, $G_{M o}$. This choice of performance index seeks to minimize the mechanical-mode oscillations of the SCG system with minimum governor valve movements. As is seen, the speed deviation, $\omega(k)$, is weighted by the elapsed time $k T$. Thus, a low value of $J$ corresponds to a small settling time, a small steady state error, and small overshoots in rotor speed, rotor angle and valve position. The performance index is minimized subject to the following constraints:

$$
\begin{aligned}
& G_{S, \text { min }} \leq G_{S} \leq G_{S, \text { max }} \\
& T_{1, \text { min }} \leq T_{1} \leq T_{1, \text { max }} \\
& T_{2, \text { min }} \leq T_{2} \leq T_{2, \text { max }} \\
& K_{v, \text { min }} \leq K_{v} \leq K_{v, \text { max }} \\
& T_{3, \text { min }} \leq T_{3} \leq T_{3, \text { max }} \\
& T_{4, \text { min }} \leq T_{4} \leq T_{4, \text { max }}
\end{aligned}
$$

The PSO algorithm iteratively updates the velocity of each particle using its current velocity and its distance from "global best position" $\left(g_{\text {best }}\right)$ and "personal best position" $\left(p_{\text {best }}\right)$ according to the following equation:

$$
\begin{aligned}
v_{i}^{k}= & w^{k} v_{i}^{k-1}+c_{1} r_{1}\left(p_{\text {best }, i}-x_{i}^{k-1}\right) \\
& +c_{2} r_{2}\left(g_{\text {best }, i}-x_{i}^{k-1}\right)
\end{aligned}
$$

where:

$i=1,2,3, \ldots, \mathrm{m}$

$v_{i}^{k}$ is the velocity of particle $i$ at iteration $k$

$x_{i}^{k}$ is the position of particle $i$ at iteration $k$

$r_{1}, \quad r_{2}$ are uniformly distributed random numbers in the range [0-1]

$c_{1}, c_{2}$ are positive constants

$w^{k}$ is the inertia weight at iteration $k$, decreasing as $w^{k}=\alpha w^{k}$.

$\mathrm{m}$ is the number of particles in a swarm, and $\alpha$ is a decrement constant.

PSO itself has a number of parameters to be properly specified. The main PSO parameters are the initial inertia weight, $w^{0}$, and the maximum allowable velocity, $V_{\max } . w^{0}$ is set at 1 , and $V_{\max }$ at $12.5 \%$ of the search space for each tuning parameters. The swarm size is chosen to be 60 particles. Other parameters are set as decrement constant $\alpha=0.98$, and $c_{1}=c_{2}$ $=2$.

\section{Simulation Results}

The author examined a number of alternatives in developing the proposed scheme. The performance index was evaluated, in all cases, in response to a three-phase to ground fault of 120 -ms duration with the operating point ( $P_{t}=0.8$ p.u, $Q_{t}=0.6$ p.u). The first attempt was the individual design for the SVC-based stabilizer; considering no governor controller, i.e. $u=0$. Then, the optimal set of $\left(K_{v}, T_{3}, T_{4}\right)$ for SVC-based stabilizer was searched for; considering governor controller with $G_{\mathrm{s}}=0.1$ $T_{1}=0.5 \mathrm{~s}$ and $T_{2}=0.01 \mathrm{~s} \quad[8,22]$. Finally, coordinated design for best combination of $\left(G_{\mathrm{s}}\right.$ $\left., T_{1}, T_{2}\right)$ for $\mathrm{GC}$, and $\left(K_{v}, T_{3}, T_{4}\right)$ for SVCbased stabilizer was sought.

Variation of the performance index $J$ with the number of iterations at different seed values is shown in Fig. 4. The optimal coordinated values selected by PSO for $\left(G_{\mathrm{s}}, T_{1}, T_{2}\right)$ and $\left(K_{v}\right.$ $\left., T_{3}, T_{4}\right)$ are $(0.065,1,0.01)$ and $(1.142,0.183$, $0.063)$ respectively.

Performance of the SCG system with the proposed control scheme following a 3-phase short circuit fault, at $\left[\left(P_{t}, Q_{t}\right)=(0.8,0.6),(0.9\right.$, $0),(0.7,-0.2)$ p.u] is shown in Figs. 5 to 7. Figures 8, 9 and 10 show the system response to a temporary (100-ms long) $10 \%$ step increase in the governor set point at the previous loading conditions. 


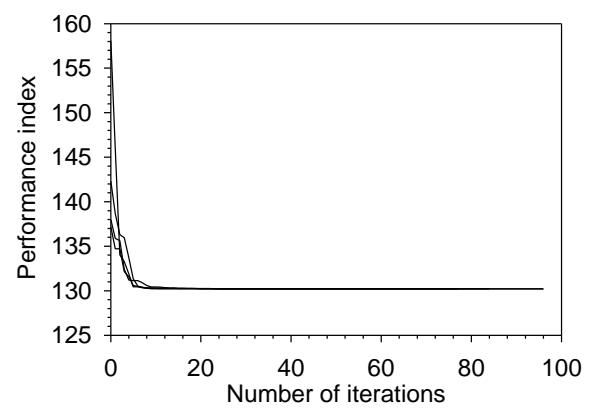

Fig. 4 Convergence of performance index with iterations at different seed values
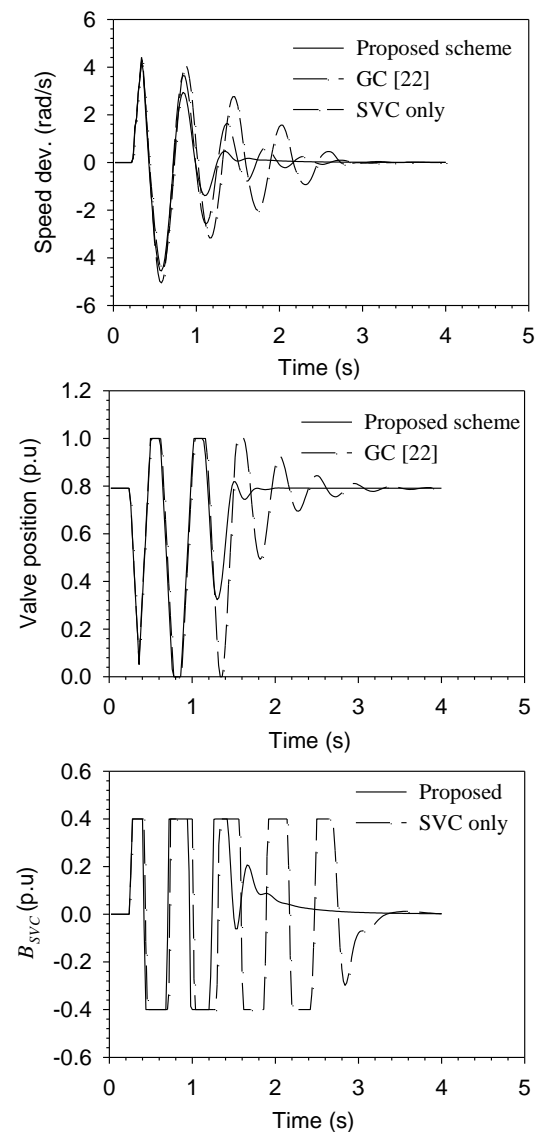

Fig. 5 Response to a 3-phase $\mathrm{SC}$ at $\mathrm{P}_{\mathrm{t}}=0.8 \mathrm{pu}, \mathrm{Q}_{\mathrm{t}}=0.6 \mathrm{pu}$
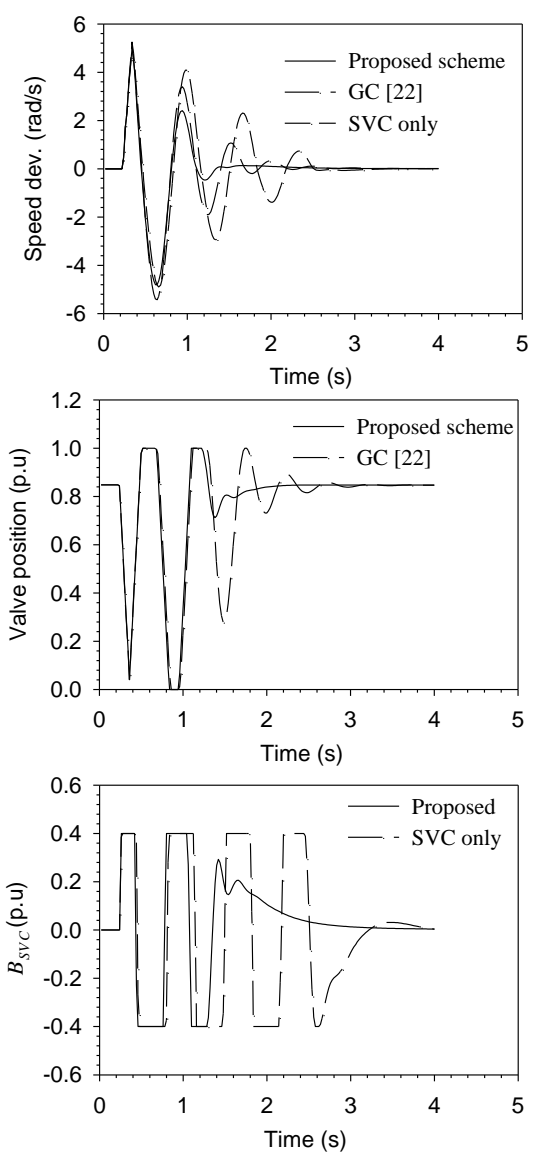

Fig. 6 Response to a 3-phase $S C$ at $P_{t}=0.9 p u, Q_{t}=0$ pu
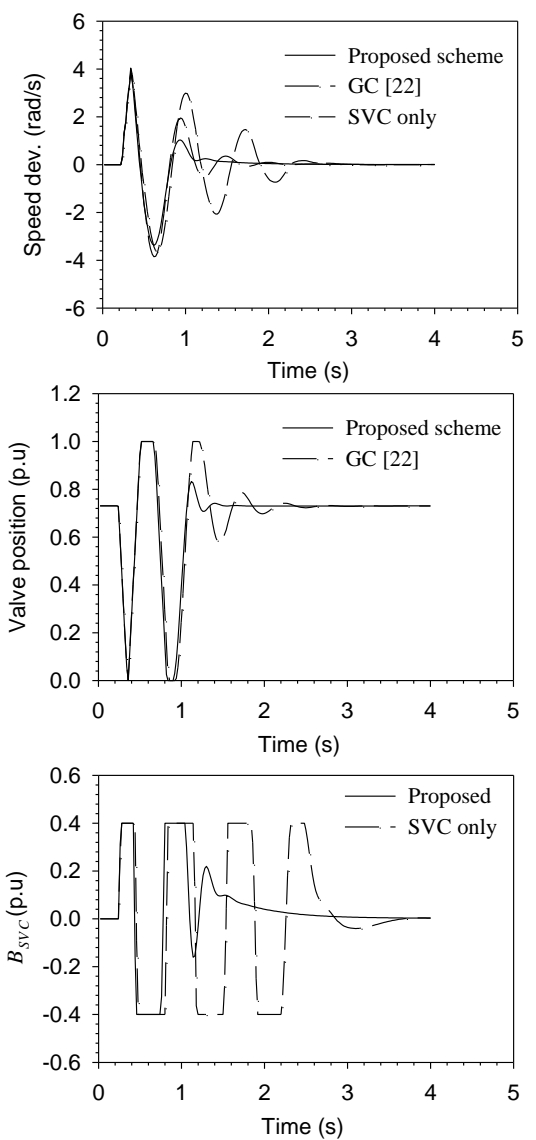

Fig. 7 Response to a 3-phase $S C$ at $P_{t}=0.7 p u, Q_{t}=-0.2$ pu 


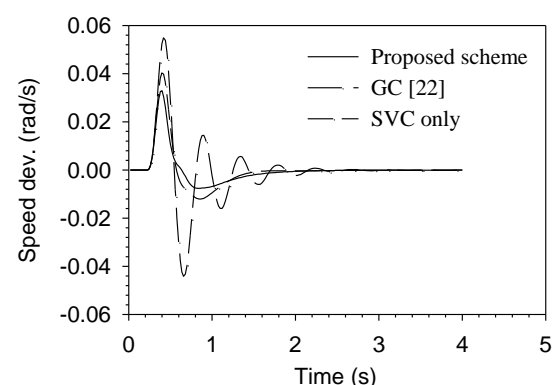

Fig.8 Response to a $10 \%$ pulse in $\mathrm{U}_{\mathrm{gr}}$ at $\mathrm{P}_{\mathrm{t}}=0.8 \mathrm{pu}, \mathrm{Q}_{\mathrm{t}}=0.6 \mathrm{pu}$

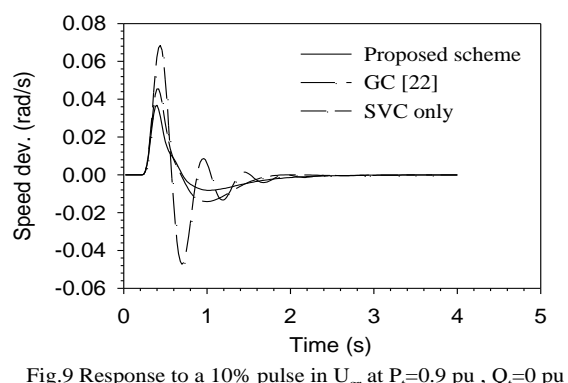

The results show that the proposed control scheme results in a significant improvement in the SCG transient performance and a considerable reduction in the rotor oscillations with acceptable valve movements.

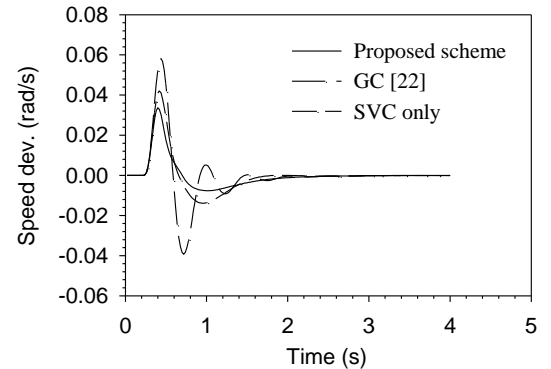

Fig.10 Response to a $10 \%$ pulse in $\mathrm{U}_{\mathrm{gr}}$ at $\mathrm{P}_{\mathrm{t}}=0.7 \mathrm{pu}, \mathrm{Q}_{\mathrm{t}}=-0.2 \mathrm{pu}$

\section{Damping and Synchronizing Torques Analysis}

The object of this section is to investigate the effects of the proposed control scheme and other schemes on the SCG dynamic performance using the concept of damping and synchronizing torques, which was initially introduced by Demello and Concordia [23]. This concept indicates that, at any given frequency of rotor oscillations, there exists oscillatory electrical torque acting on the rotor which has the same frequency and whose amplitude is proportional to the amplitude of the oscillations. The change in this torque $\Delta T_{e}$ can be divided into two components: one is in time phase with, and proportional to the rotor angle deviation $\Delta \delta$. This is called the "synchronizing torque". The other, which is in time phase with and proportional to the rotor speed deviation $\omega$ is called the "damping torque". Therefore, the change in electrical torque can be written as follows:

$$
\Delta T_{e}=K_{s} \Delta \delta+K_{d} \omega
$$

where $K_{s}$ and $K_{d}$ are the synchronizing and damping coefficients respectively. It is now well recognized that machine stability is highly degraded if there is lack of either or both of synchronizing and damping torques. The values of $K_{s}$ and $K_{d}$ are determined from the time responses of electrical torque, rotor angle and rotor speed, using the technique explained in Ref. [24-25]. In that technique, the error between the actual torque deviation and that obtained by summing the damping and synchronizing torque components is defined as:

$$
E(t)=\Delta T_{e}(t)-\left[K_{s} \Delta \delta(t)+K_{d} \omega(t)\right]
$$

The error squares can be summed over the simulation time period. Minimizing this summation with respect to $K_{s}$ and $K_{d}$ yields the following dependent algebraic equations:

$$
\begin{aligned}
& \sum_{n} \Delta T_{e} \Delta \delta=K_{s} \sum_{n}(\Delta \delta)^{2}+K_{d} \sum_{n} \omega \Delta \delta \\
& \sum_{n} \Delta T_{e} \omega=K_{d} \sum_{n} \omega^{2}+K_{s} \sum_{n} \omega \Delta \delta
\end{aligned}
$$

Solving the equations (32) and (33) gives the values of $K_{s}$ and $K_{d}$, where $n$ is the discretesimulation time.

A summarized comparison of the proposed scheme and other schemes (viz. SVC with GC [22], and GC [22] only) is shown in Table 1.

Table 1: Comparison of the proposed scheme and other schemes

\begin{tabular}{|l|c|c|c|c|c|}
\hline \multicolumn{1}{|c|}{$\left(P_{t}, Q_{t}\right)$ p.u } & \multicolumn{3}{|c|}{$(\mathbf{0 . 8}, 0.6)$} & \multicolumn{2}{c|}{$(0.7,-0.2)$} \\
\hline $\begin{array}{l}\text { Coordinated } \\
\text { SVC with } \\
\text { GC }\end{array}$ & 130.2 & 0.231 & 1.941 & 0.212 & 1.184 \\
\hline $\begin{array}{l}\text { SVC with } \\
\text { GC [22] }\end{array}$ & 138.4 & 0.166 & 1.836 & 0.142 & 1.11 \\
\hline $\begin{array}{l}\text { GC [22] } \\
\text { only }\end{array}$ & 261.7 & 0.014 & 2.011 & 0.016 & 1.251 \\
\hline
\end{tabular}

From this table, it can be concluded that the proposed scheme outperforms the other considered schemes at all operating points studied. It provides the SCG system with the highest possible degree of damping while keeping the synchronizing torque at a high level.

\section{Small Signal Analysis}

In this section, the dynamic behaviour of the SCG system with the proposed control scheme is investigated using the eigenvalues technique to get another quantitative assessment of the damping provided by the SVC-based stabilizer with the GC. The non-linear system equations are linearized about a quiescent operating 
point. The linearized model's equations can be expressed in the state-space form as follows:

$$
[d x / d t]=[A][x]+[B][u]
$$

where $[x]$ is the state vector defined as:

$$
\begin{aligned}
& {[x]=\left[\Delta \delta, \Delta \omega, \Delta \psi_{f}, \Delta \psi_{d}, \Delta \psi_{D 1}, \Delta \psi_{D 2}, \Delta \psi_{q},\right.} \\
& \left.\Delta \psi_{Q 1}, \Delta \psi_{Q 2}, \Delta Y_{H P}, \Delta Y_{R H}, \Delta Y_{I P}, \Delta Y_{L P}, \Delta G_{M}, \Delta B\right]^{\mathrm{T}}
\end{aligned}
$$

The dynamic response of the SCG system can be determined from the eigenvalues of the matrix $[A]$. The eigenvalues related to the rotor mode of oscillation at two operating points are given in Table 2 . The results show clearly that the rotor oscillation without any stabilizer is slightly damped, its damping ratio, $\xi$, being only 0.011 at the first operating point. It is also shown that this mechanical oscillation becomes well damped when the GC is included. The damping ratio in case of the proposed scheme is about 2.3 times that with the GC alone. This confirms that the coordinated SVC-based stabilizer and GC provide more damping to the SCG rotor oscillations.

Table 2: Eigenvalues related to rotor mode of oscillation

\begin{tabular}{|l|c|c|c|}
\hline \multicolumn{1}{|c|}{$\left(\boldsymbol{P}_{\boldsymbol{t}}, \boldsymbol{Q}_{t}\right)$} & \multicolumn{2}{|c|}{$(\mathbf{0 . 8 , 0 . 6}) \mathbf{p . u}$} & $(\mathbf{0 . 7}, \mathbf{- 0 . 2}) \mathbf{p . u}$ \\
\hline & Eigenvalues & $\xi$ & Eigenvalues \\
\hline $\begin{array}{l}\text { Proposed } \\
\text { SVC with } \\
\text { GC }\end{array}$ & $-11.88 \pm \mathrm{j} 24.3$ & 0.439 & $-12.8 \pm \mathrm{j} 23.16$ \\
\hline $\begin{array}{l}\text { SVC with } \\
\text { GC [22] }\end{array}$ & $-10.97 \pm \mathrm{j} 21.46$ & 0.455 & $-11.94 \pm \mathrm{j} 20.35$ \\
\hline $\begin{array}{l}\text { GC [22] } \\
\text { only }\end{array}$ & $-2.676 \pm \mathrm{j} 13.74$ & 0.191 & $-3.526 \pm j 11.81$ \\
\hline $\begin{array}{l}\text { Without } \\
\text { stabilizers }\end{array}$ & $-0.118 \pm \mathrm{j} 11.02$ & 0.011 & $-0.216 \pm j 9.045$ \\
\hline
\end{tabular}

\section{Conclusion}

This study described the utilization of one of FACTS devices for stability enhancement of superconducting generators. An approach was proposed for the design of a static VAR compensator-based stabilizer in coordination with a governor controller to provide more damping to mechanical oscillations of the SCG studied. A performance index was defined and the PSO technique was used to select the optimal parameters of both SVC-based stabilizer and GC. Simulation results show the effectiveness of the proposed control scheme in damping the rotor oscillations, and enhancing the SCG stability over a range of operating conditions and various disturbances. Analysis of damping and synchronizing torques, and small signal analysis were used to provide other quantitative assessments of the SCG performance with the designed SVCbased stabilizer and GC. Results of these analyses verify the effectiveness of the proposed approach with the SCG system studied.

\section{References}

[1] N. Maki, K. Yamaguchi, M. Takahashi, and R. Shiobara, "Development of superconducting AC generator," IEEE Trans. on Magnetics, Vol. 24, No. 2, pp. 792795, 1988

[2] T. Nitta, Y. Shirai, T. Kawauchi, T. Okada, and Y. Ogawa, "Transient stability limit issues at three-phase short-circuit in parallel running of both a superconducting generator and a conventional one", Electrical Engineering in Japan, Vol. 115, No. 6, pp. 62-70, 1995.

[3] K. Ueda, R. Shiobara, M. Takahashi, and T. Ageta, "Measurement and analysis of 70 MW superconducting generator constants", IEEE Trans. on Applied Superconductivity, Vol.9, No.2, pp.11931196, 1999.

[4] H. Tsukiji, T. Hoshino, and I. Muta, "Output power limit of $200 \mathrm{MW}$ class brushless super-conducting generator excited with magnetic flux pump" IEEE Trans. on Applied Super-conductivity, Vol. 11, No.1, pp.2335-2338, 2001.

[5]http://events.energetics.com/wire05/, K. Amm, "100 MVA HTS Generator Development Update", DOE HTS Wire Workshop, January 19-20, 2005.

[6] M. Maki, "Design study of hightemperature superconducting generators for wind power systems", 2008, Journal of Physics: Conf. Ser. 97012155 (6pp)

[7] K. F. Goddard, B. Lukasik, and J.K. Sykulski, "Alternative Designs of High-Temperature

\section{Superconducting Synchronous}

Generators", IEEE Trans. on Applied Superconductivity, Vol. 19, No.6, pp. 3805-3811, 2010.

[8] M. A. A. S. Alyan and Y. H. Rahim, "The role of governor control in transient stability of superconducting turbogenerators," IEEE Trans. on EC, Vol. 2, No. 1, pp. 38-46, 1987.

[9] S.M. Osheba, M.A.A.S. Alyan, and Y.H.A. Rahim, "Comparison of transient performance of superconducting and 
conventional generators in a multimachine system", IEE Proc., Pt. C, Vol. 135, No.5, pp. 389-395, 1988.

[10] R.A.F. Saleh, "Application of artificial intelligence techniques to the design of improved stabilizers for superconducting generators", Ph.D. thesis, Cardiff University, Cardiff, UK, 2001.

[11] N.G. Hingorani, "High power electronics and flexible AC transmission system", IEEE Power Engineering Review, July 1988.

[12] A. Edris, "FACTS technology development: an update", IEEE Power Engineering Review, pp.4-9, March 2000.

[13] M. Noroozian and G. Anderson, "Damping of power system oscillations by use of controllable components", IEEE Trans. PWRD, Vol.9, No.4, pp.20462054, 1994.

[14] M. A. Abido and Y. L. Abdel-Magid, "Coordinated design of a PSS and an SVC-based controller to enhance power system stability", International Journal of Electrical Power and Energy Systems, Vol.25, No.9, pp. 695-704, 2003.

[15] R.M. Mathur, P.K. Dash and A.E. Hammad, "Transient and small signal stability of a superconducting turbogenerator operating with thyristor controlled static compensator", IEEE Trans. on PAS, Vol.98, No.6, pp.19371946, 1979.

[16] A. A. Abou El-Ela, T. Fetouh, M. A. Bishr and R.A.F. Saleh, "Power systems operation using particle swarm optimization technique", Electric Power Systems Research 78, pp. 1906-1913, 2008.

[17] P. J. Lawrenson, T. J. E. Miller, J. M. Stephenson, and A. H. M. S. Ula, "Damping and screening in the synchronous superconducting generator," Proc. IEE, Vol. 123, No. 8, pp. 787-794, 1976.

[18] B.W. Hogg, "Representation and control of turbogenerators in electric power systems", Chapter 5 in 'Modelling of dynamical systems', Vol.2, Peter Peregrinus Ltd., 1981.

[19] M. Pavella, and P.G. Murthy, "Transient stability of power systems: theory and practice", John Wiley \& Sons, 1st edition, 1994.

[20] R. Eberhart, and Y. Shi, "Particle swarm optimization: developments, applications and resources", Proc. of the 2001 Congress on Evolutionary Computation, Vol. 1, pp. 81-86, 2001.

[21] S. Lefebvre, "Tuning of stabilizers in multi-machine power systems", IEEE Trans. on Power Apparatus and Systems, Vol. 102, No. 2, pp. 290-299, 1983.

[22] G.A. Morsy, A. Kinawy, and S.M. Osheba, "Frequency domain analysis of a superconducting generator", Electric Power Systems Research, No. 30, pp. 107113, 1994.

[23] F. P. Demello, and C. Concordia, "Concepts of synchronous machine stability as affected by excitation control", IEEE Trans. on Power Apparatus and Systems, Vol. 88, No. 4, pp.316-329, 1969.

[24] R.T.H. Alden, and A.A. Shaltout, "Analysis of damping and synchronizing torques", IEEE Trans. on Power Apparatus and Systems, Vol. 98, No. 5, pp.1696-1700, 1979.

[25] F.M. Abdel-Kader, and S.M. Osheba, "Performance analysis of permanent magnet synchronous motors", IEEE Trans. on Energy Conversion, Vol. 5, No. 2, pp.366-373, 1990.

\section{Appendix A}

The parameters of the SCG system used in this study (inductance and resistance values in p.u; time constants in seconds) are $[8,9]$ :

Superconducting generator parameters:

$L_{f}=0.541, L_{d}=L_{q}=0.5435, L_{D I}=L_{Q 1}=0.2567$,

$L_{D 2}=L_{Q 2}=0.4225$

$L_{f d}=L_{f D 1}=L_{d D 1}=L_{d D 2}=L_{D 1 D 2}=0.237$

$L_{f D 2}=0.3898, L_{q Q 1}=L_{q Q 2}=L_{Q 1 Q 2}=0.237$

$\tau_{\mathrm{f}}=750, R_{d}=R_{q}=0.003$

$R_{D I}=R_{Q 1}=0.01008, R_{D 2}=R_{Q 2}=0.00134$

$H=3 \mathrm{~kW} . \mathrm{s} / \mathrm{kVA}$

Transformer and transmission line parameters: $X_{T}=0.15, R_{T}=0.003, X_{L}=0.05, R_{L}=0.005$

Turbine and governor parameters:

$\tau_{G M}=\tau_{G I}=0.1, \tau_{H P}=0.1, \tau_{R H}=10$

$\tau_{I P}=\tau_{L P}=0.3, P_{o}=1.2$ p.u.

$F_{H P}=0.26, F_{I P}=0.42, F_{L P}=0.32$

Valve position and movement constraints are defined by:

$0 \leq\left(G_{M}, G_{I}\right) \leq 1$ and $-6.7 \leq\left(p G_{M}, p G_{I}\right) \leq 6.7$ 